\title{
RADIO-COMMUNICATIONS SYSTEMS IN INDOOR ENVIRONMEN'TS: PERFORMANCES OF THE CHANNEL EQUALIZATION
}

\author{
Fernando J. CASADEVALL \\ Universitat Politécnica de Catalunya (UPC) \\ Apdo. 30.002, 08080 Barcelona (spain)
}

\begin{abstract}
In thls paper, the periormances of M-QAM Indoor radio systems that use diversity and equalization techniques are studied. The system performances are described in terms of the Outage Probability.
\end{abstract}

\section{INTRODUCTION}

The use of radio in indoor data communications is an attractive proposition because it gives total mobility to the increasing number of terminal equipments in large buildings. However, indoor radio systems are affected by frequency selective fading caused by the multipath time delay spread that produces intersymbol interference (ISI), thus resulting in an irreducible bit error rate (BER) and imposing an upper limit on the data symbol rate. In order to combat this situation, diversity techniques and or adaptive channel equalizers could be used. In this paper, we assess the performances of M-QAM indoor radio systems that use diversity and equalization techniques. 4QAM and 16-QAM have been considered. The influences on the system performances of various system and channel parameters, like the r.m.s. delay spread of the Power Delay Profile that characterize the propagation conditions, the equalizer structure (linear and non-linear) as well as the number of taps etc... have been taken into account. The system performances are described in terms of the Outage Probability.

\section{TRANSMISSION MODEL}

Figure 1 shows the low-pass equivalent model of the transmission system. The transmitted signal can be formulated as:

$$
\begin{aligned}
s(t) & =\sum_{k=-\infty}^{\infty}\left(a_{k}+j b_{k}\right) \cdot \delta(t-k T) \\
& =\sum_{k=-\infty}^{\infty} d_{k} \cdot \delta(t-K T)
\end{aligned}
$$

where $\left\{a_{k}\right\},\left\{b_{k}\right\}$ are data sequences of duration $T$ for the inphase and quadrature channels. They are $\pm 1, \pm 3, \ldots$, $\pm\left(M^{1 / 2}-1\right)$ with $M=4$ for 4-QAM and $M=16$ for 16-QAM. Moreover, $a_{k}$ and $b_{k}$ are independent random variables. The overall filtering transfer function $\mathrm{H}_{T}(i) \cdot \mathrm{H}_{\mathrm{R}}(\mathrm{f})$ is a raisedcosine type with a roll-off factor equal to 0.5 . The filtering is split equally between the transmitter and the receiver. $h_{c}(t)$ models the channel behavior that introduces selective fading in the radio link. The channel is assumed to be wide sense stationary uncorrelated scattering (WSSUS) and it is represented by a unique correlation function referred to as the Power Delay Profile, $P(t),[1]$. A measure of the width of $P(t)$ is the root mean-square delay spread, $\tau$. From the Power Delay Profile function, a sample of the channel impulse response can be constructed by the following formula :

$$
h_{c, 1}(t)=\sqrt{t_{n}} \cdot \sum_{n=1}^{L}\left(h_{1 n, 1}+j h_{c n, 1}\right) .8\left(t-t_{n}\right)
$$

where $i=1,2, h_{i}, h_{q n}$ are zero mean gaussian random variables with variance $P\left(n . t_{n}\right) / 2$ and $t_{n}$ is the time between samples. The number of samples, $L$, needed to represent the indoor mobile channel in an accurate form, depends on the shape of the Power Delay Profile, $P(t)$, and on its rms delay spread. Measurements from many different buildings, [1] allow us to consider that the most common shape for the Power Delay Profile is the one-side exponential profile, given by:

$$
P(t)= \begin{cases}\frac{1}{\tau} \cdot \exp \left[-\frac{t}{\tau}\right] & t \geq 0 \\ 0 & t<0\end{cases}
$$

In this case $t_{n}=\tau / 2$ and it is sufficient to consider a time duration of the one-side exponential profile approximately equal to $14 \tau$.

The received signal $r_{1}(t)$ (also $r_{2}(t)$ if diversity technique is considered) can be expressed by: 


$$
\begin{aligned}
I_{i}(t) & =\sum_{k=-\infty}^{\infty}\left[a_{k} \cdot h_{i}^{R}(t-K T)-b_{k} \cdot h_{i}^{T}(t-k T)\right]+ \\
& +j \sum_{k=-\infty}^{\infty}\left[b_{k} \cdot h_{i}^{R}(t-K T)+a_{k} \cdot h_{i}^{T}(t-K T)\right]+ \\
& +n_{x}(t)+j n_{y}(t)
\end{aligned}
$$

where, in generai

$$
\begin{aligned}
h_{1}(t) & =h_{1}^{R}+j h_{1}^{I}= \\
& =\left[F^{-1}\left[H_{T}(f) \cdot H_{R}(f)\right] * h_{c, 1}(t)\right] \cdot G_{1} \cdot e^{-j 0}
\end{aligned}
$$

with $=1,2 . F^{-1}$ denotes the inverse Fourier transform, ${ }^{\bullet}$ is the convolution operator, and $\mathrm{G}_{\mathrm{i}}$ is a gain factor introduced to considered the presence of automatic gain control (CAG). We have taken into account a carrier recovery circuit that minimizes the output mean square error,[3]. Assuming that the bandwidth of the carrier recovery circuit is much higher than the fading rate, it could be considered that the carrier phase can be tracked as if the global impulse response is time-invariant. As we focus on the effects of the delay spread, the phase jitter on the recovered carrier caused by the gaussian noise will not be taken into account. The optimum sampling instant is obtained from a classical squaring timing recovery loop. [4]. Two baseband equalizer structures have been analyzed. Linear and Non-Linear equalizers both with baud period $T$ spacing between stages. In all the cases, the minimum mean-square error (MMSE) technique has been adopted to calculate the tap values.

\section{RESULTS}

The objective of this paper is to perform a comparative study of the different equalizer structures for high level M-QAM modulations and to determine the data rate limitation for indoor communication systems. The criterium used to evaluate the system quality is the outage probability, defined as :

$$
P_{0}=\operatorname{Prob} .\left(P_{0}>10^{-\gamma}\right)
$$

where $P_{e}$ is the error probability and $\gamma$ is a constant that we have taken equal to 2 or 6 . To compute the error probability integral we consider the Levi's method, [5].

In figure 2 the evolution of the outage probability, against the normalized delay spread of the Power Delay Profile, called $\tau / T$, is shown. It can be seen that a system without any protection has a very limited performances since for $\tau \pi>0.1$ the outage probability is greater than 0.1 (10\%). Moreover, for smaller values of $\tau / T$, the outage probability goes to an asymptotic value (marked by the letter $A$ on the figures) that is greater than $0.01(1 \%)$. It is important to emphasize that this value shows the behavior of the system periormances when a flat fading channel is considered. If the diversity technique is considered we can see that for high values of the ratio $\tau / T$ the system pertormances are only slightly better than the obtained for a system without protection, but when smaller values are taken into account the values of the outage probability converge on the value for a flat fading channel. On the other hand, for small values of the normalized delay spread, the system performances increase by a factor of ten approximately. In conclusion, the diversity technique could be used in an effective way if a flat channels or channels with low distortion are considered. When base band equalizers are considered the system performances remain very limited. For low values of $\tau / T$, the outage probability goes to the asymptotic value corresponding to a system without any protection. This could be explained considering that if $z / T<0.1$ the $|S|$ is very small and the system performances become limited by the Rayleigh nature of the propagation. However for higher values of $\tau / T$ the improvement in the system performances due to use equalization techniques are more significative. Certainly for a non-linear equalizer with 4 or 5 taps (marked as $1+2$ or $1+3$ in the figure 2) the outage probability is close to the asymptotic value even for very distorted channels as $\tau / T=$ 0.5 . However, considering only equalization techniques the outage probability can not be lower than $4.10^{-2}$. Then the system performances are very limited and more powerful techniques must be considered.

II joint equalizer and diversity techniques are taken into account, figures 3 and 4 , the performance of the system increases quickly. For a BER of $10^{-2}$,SNR equal to $20 \mathrm{~dB}$ and a linear equalizer of 5 taps in each branch (marked as $2+2$ in the figures) the system is able to guarantee an outage probability lower than $0.001(0.1 \%)$ for values of the normalized delay spread smaller than 0.8 . On the other hand, we can see that a non-linear oqualizer is able to guarantee an outage probability lower than $0.001(0.1 \%)$ even for values of $\tau / T=1$, that is even if the channel shows a high distortion. If a BER value of $10^{-6}$ is considered the outage probability is approximately equal to $0.01(1 \%)$ for values of the normalized delay spread lower than 0.6. Notice that a linear equalizer gives poor pertormances for high values of the delay spread. In order to obtain an outage probability lower than $10^{-3}$ for BER values of $10^{-6} \mathrm{a}$ higher signal to noise ratio must be considered. It is important to emphasize that, when joint equalization and diversity techniques are considered, the system performances are better than the obtained for a flat fading 
channel for values of $\tau / T$ ranging from 0.01 to 0.5 . This could be explained because the system uses the multipath as a additional redundant channels to increase the diversity gain. However, for lange values of $\tau \pi$ the induced intersymbol interference ,due to the multipath, increases considerably and the equalizer can not cope completely with it, and as the result the system performances degrades quickly.

Figures 5 and 6 show the system performances when 16 QAM modulation is considered. In this case a signal to noise ratio of $30 \mathrm{~dB}$ is taken into account. The behavior of the linear and non linear equalizer is shown considering 3 and 5 taps in each diversity branch (marked in the figure by $1+1$ and $2+2$ respectively). For a BER of $10^{-2}$ a linear equalizer could guaranteed an outage probability lower than $0.01(1 \%)$ for values of $\tau /<0.3$ if 3 taps are taken into account, and $\tau / T<0.5$ when 5 taps are used. An outage probability lower than $10^{-3}$ could only be guaranteed for $\tau / T \equiv 0.1$. However if a nonlinear equalizer with 5 taps (marked by 2+2) is considered the outage probability could be lower than $10^{-3}$ if $\tau / T<0.55$ and lower than $10^{-2}$ if $\tau / T<0.7$. For a BER of $10^{-6}$ a non linear equalizer with 5 taps is necessary in order to obtain an outage probability lower than $10^{-2}$ for $\tau / \pi<0.4$

\section{CONCLUSIONS}

We have analyzed the performances of the diversity and equalization techniques in an indoor radio environment. 4 and 16 modulation have been considered. From the obtained results we can conclude that a system without protection has very limited performances. When the channel introduces low level of distortion, the diversity technique produces better performances than the equalizer technique, but if the channel introduces a high degree of intersymbol interference, then the equalizer techniques are slightly better than the diversity techniques. On the other hand the joint equalization and diversity techniques are a very effective tool to combat the degrading effect introduced by the indoor channel. improvement in the system performances, with respect to a system without any protection, ranging from 10 to 100 have been obtained.

\section{REFERENCES}

[1] J.G. PROAKIS: Digital Communications, Mc Graw Hill, 1983. Chapter 7

[2] J.C.CHUANG: The effects of Time Delay Spread on Portable Radio Communications Channels with Digital Modulation, IEEE J. Selected Areas on Communication, vol. SAC-5, N25,pp. 879-889, June 1987

[3] S. MORIDI, H. SARI: Analysis of DecisionFeedback Carrier Recovery Loops with applications to 16 QAM Digital Radio Systems. International Conference on Communications (ICC'83), 1983,pp. 671-675.

[4] N. AMITAY, L.J. GREENSTEIN, "Multipath Outage Performance of Digital Radio Receivers Using Finite-taps Adaptive Equalizers." "IEEE Trans. on Communications, Vol. COM-32, № 5, May 1984.

[5] A. LEVI,"Fast Error Rate Evaluation in the Presence of Intersymbol Interference".IEEE Trans. on Communications, Vol. COM-33, N25, May 1985, pp. 479-481

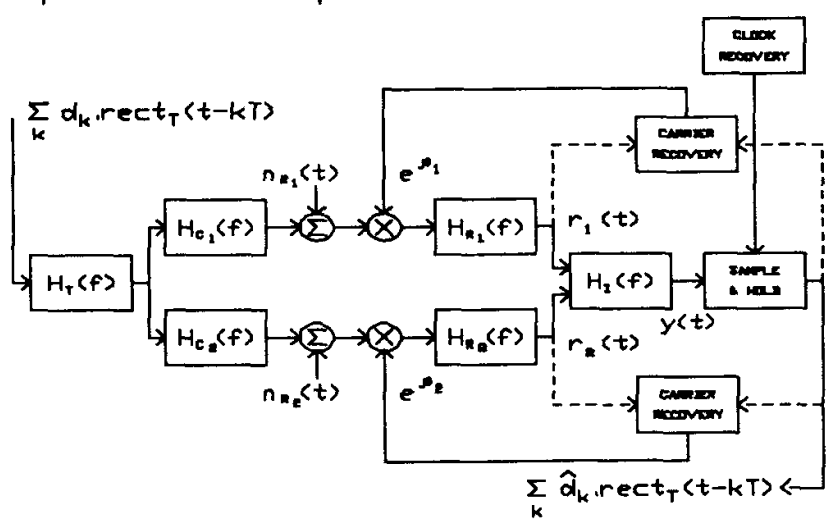

Figure 1.- LOW PASS EQUIVALENT MODEL OF THE TRANSMISSION SYSTEM 


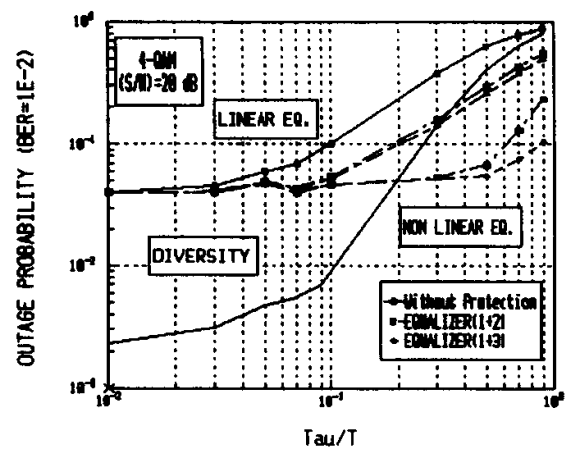

Figuire 2. -ouTAGE PROBABILITY VERSUS MORMALIZED DELAY SPREAD.

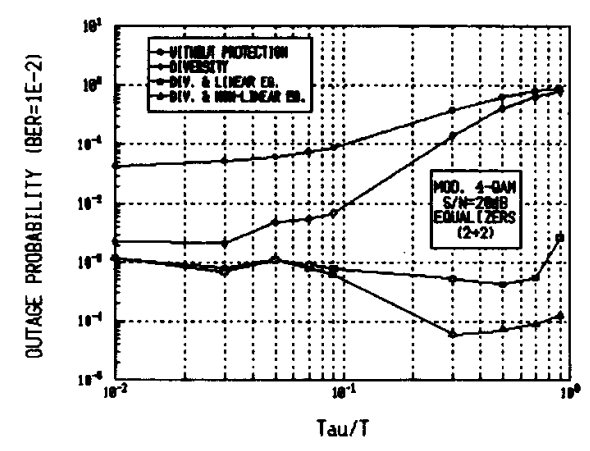

Figurce 3. -aUtAGE PROBABILITY VERSUS HORMALIZED DELAY SPREAD.

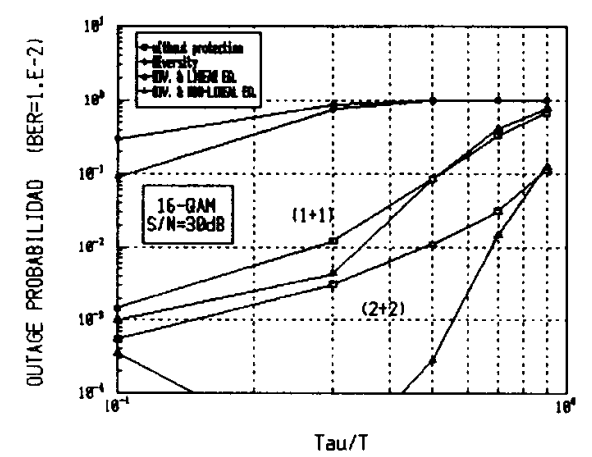

Figure 5. - OUTAGE PROBABILITY VERSUS NORMAL IZED DELAY SPREAD

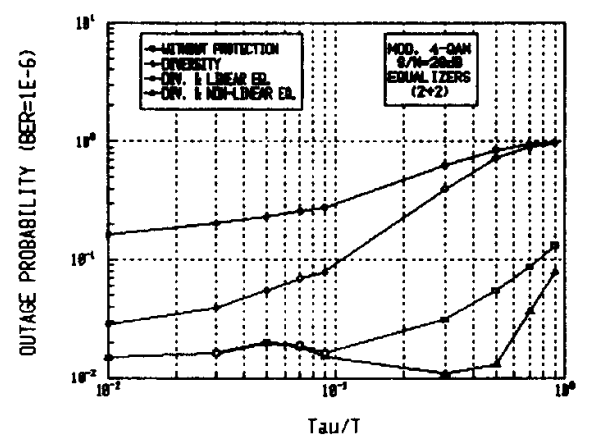

Figure 4. -OUTAGE PROBABILITY VERSUS MORMALIZED DELAY SPREAD

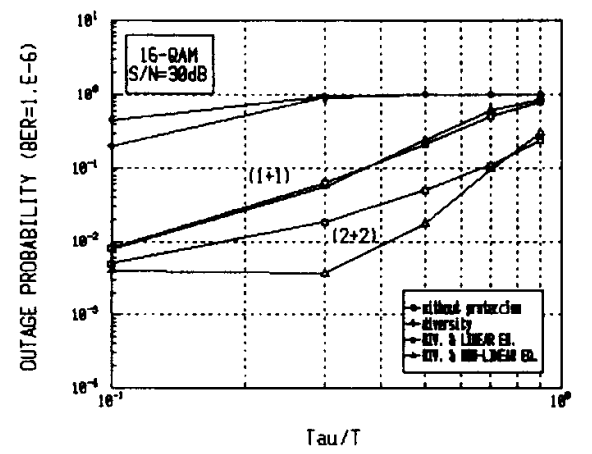

Figure 6. - OUTAGE PROBABI LITY VERSUS MORMALIZED DELAY SPREAD

\section{ACRERTIENTS}

This work has been financed by Spain CICYT TIC880543. 